\title{
No Difference in Outcomes Between Suspensory (Fixed-Loop Cortical Button) Versus Expandable Anteromedial Femoral Fixation in Anterior Cruciate Ligament Reconstruction With Autologous Hamstring Tendons: A Prospective, Randomized, Controlled Study in Male Patients
}

\author{
Andreas Panagopoulos, M.D., Ph.D, Dimitris Mylonas, M.D., Antonis Kouzelis, M.D., Ph.D., \\ Petros Zampakis, M.D., Ph.D., Pantelis Kraniotis, M.D., John Lakoumentas, Ph.D., and \\ John Gliatis, M.D., Ph.D.
}

\begin{abstract}
Purpose: To compare anterior cruciate ligament (ACL) autograft reconstruction using fixed-looped suspensory versus expandable femoral fixation through the anteromedial portal. Methods: After we performed prospective power analysis and obtained institutional review board, 70 patients with ACL rupture were block randomized to the expandable or suspensory femoral fixation group (35 in each group). All patients received autologous hamstring autograft through the anteromedial portal and fixed with a sheath-screw system in the tibia. The primary outcome measures were anteroposterior knee stability at 2 years' follow-up measured using the KT-1000 arthrometer and the degree of femoral and tibial tunnels' widening measured by the use of computed tomography imaging performed immediately postoperative and 12 months postsurgery. Secondary outcome measures included pain score on a visual analog scale, the subjective International Knee Documentation Committee 2000 assessment form, the Lysholm score, and the Tegner activity scale at 3, 6, 12, and 24 months. Results: Twenty-four patients were excluded from further analysis, leaving a total of 48 patients (24 in each group) for the final evaluation. The anteroposterior knee stability (KT-1000) showed no difference between groups at 24 months' follow-up $(P=.31)$. The percentile widening $(\%)$ of femoral and tibial tunnel at l-year follow-up showed no difference also, except for greater values at the tibial coronal point T2 $(P=.065)$ and tibial sagittal point $\mathrm{Tl}$ $(P=.033)$ in the group of cortical buttons. Secondary clinical outcomes showed no statistical differences between groups at $3,6,12$, and 24 months' postoperatively. Numerical pain scale (visual analog scale) was similar in both groups except for postoperative day 7 , where the AperFix group showed better results $(P=.014)$. There were no major intraoperative and late postoperative complications in any of the groups. Conclusions: Our results showed no significant differences in knee anteroposterior stability, tunnel enlargement or other clinical outcomes comparing expandable versus cortical button fixation in anteromedial hamstrings ACL reconstruction. Level of Evidence: Level II, randomized controlled trial.
\end{abstract}

$\mathbf{T}$ The influence of femoral tunnel position (anatomical or not) and the type of fixation (suspensory, aperture, intra-tunnel transfixation) on both tunnel widening and clinical outcomes in patients undergoing anterior cruciate ligament (ACL) reconstruction with

From the Departments of Sports Medicine (A.P., D.M., A.K., J.G.) and Radiology (P.Z., P.K.), Patras University Hospital Greece; and Department of Medical Physics, School of Medicine, University of Patras (J.L.), Patras, Greece.

The authors report that they have no conflicts of interest in the authorship and publication of this article. Full ICMJE author disclosure forms are available for this article online, as supplementary material.

Received December 18, 2020; accepted May 12, 2021 hamstrings autograft is still questionable and an ongoing source of debate. ${ }^{1-5}$ Several systematic reviews and meta-analyses ${ }^{6-12}$ have demonstrated either similar results $^{6-9}$ in terms of stability, clinical performance, and revision rates or better overall outcomes and less tunnel

Address correspondence to Andreas Panagopoulos, M.D., Ph.D., Patras University Hospital, Papanikolaoul, Rio-Patras, 26504, Greece. E-mail: andpan21@gmail.com

(C) 2021 THE AUTHORS. Published by Elsevier Inc. on behalf of the Arthroscopy Association of North America. This is an open access article under the CC BY-NC-ND license (http://creativecommons.org/licenses/by-nc-nd/4.0/). 2666-061X/201978

https://doi.org/10.1016/j.asmr.2021.05.003 
enlargement with suspensory, ${ }^{10}$ aperture, ${ }^{11}$ or intratunnel transfixation methods. ${ }^{12}$ Expandable femoral fixation devices ${ }^{13-20}$ recently have been introduced to theoretically provide a stronger aperture fixation with (1) circumferential compression of the hamstring's graft at both the femoral and tibial tunnels and (2) a theoretical "double-band ACL equivalent through a single tunnel." 19 In comparison with other fixation techniques for hamstring's autografts, the expandable systems have shown either similar ${ }^{16-18}$ or superior clinical results ${ }^{20}$ and in some cases less tunnel enlargement, $^{21}$ especially when they are inserted through the anteromedial portal.

The purpose of the study was to compare ACL autograft reconstruction using fixed-looped suspensory versus expandable femoral fixation through the anteromedial portal. Our hypothesis was that ACL reconstruction using expandable fixation would result in better anteroposterior stability, less tunnel widening, and probably better clinical outcome at 2 years' follow-up.

\section{Methods}

An institutional review board approval (no. 15245/ 20.07.2016-77/15.04.2016) was obtained before the initiation of the study, and all patients conceded to participate retaining every right to withdraw from the study at any time, for any reason and without any effect on their medical care. Written consent was also obtained for radiologic examination (computed tomography [CT] scan) postoperative and 1-year postoperatively (to evaluate tunnel enlargement) as well as for the use of their clinical data and intraoperative pictures for publication purposes. Seventy male patients with ACL injury participated in the study from October 2016 until September 2017. Block randomization was conducted by assigning an equal number of suspensory $(\mathrm{n}=35)$ and expandable $(n=35)$ fixation patients from the waiting list of 3 different qualified surgeons using MiniTab software (version 16.2.4; MiniTab, State College, PA). The tibial stabilization technique was the same for all patients (tibial sheath and screw and additional metal staple).

\section{Inclusion-Exclusion Criteria and Final Cohort}

The inclusion criteria were male patients aged 15 to 45 years, suffering from a complete ACL rupture who elected to undergo ACL reconstructive surgery with hamstring autograft. Exclusion criteria included (1) severe osteoarthritis (Kellgren-Lawrence grade 3 or 4 ) on standing plain radiographs; (2) injury to multiple structures of the knee (such as collateral ligaments, posterior cruciate ligament, medial patellofemoral ligament); (3) meniscal tears treated by suturing; (4) significant cartilage effects that required special treatment in addition to arthroscopic saving; (5) recent injuries $(<3$ weeks $)$ due to increased risk of fibrosis and inflammation; (6) patients with preoperative stiffness, edema, or muscle deficits or previous knee injuries (fracture, patellar dislocation) or any other knee procedure besides diagnostic arthroscopic surgery; (7) hamstrings grafts with insufficient diameter $(<7.5 \mathrm{~mm})$ that required additional synthetic or other type of graft reinforcement; and (8) inability to read and understand Greek. Twenty-two patients were excluded after their inclusion in the study; 7 refused to undergo the follow up CT evaluation, 6 underwent meniscus suturing although there was no evidence of tearing in their preoperative MRI, 3 underwent microfracturing and 1 autologous matrix-induced chondrogenesis due to medium-sized focal cartilage lesions, 3 had graft diameter $<7.5 \mathrm{~mm}$ and received synthetic graft augmentation, 1 patient left voluntarily before completing the study protocol, and another one suffered a severe knee injury one month postoperatively, as a result of a traffic accident, leaving a total of 48 patients (24 in each group) for the final evaluation.

\section{Outcome Measures}

Patient demographic data, including age and sex, occupation, athletic performance, current health, type of injury, and current knee status were collected preoperatively using the International Knee Documentation Committee (IKDC) forms. Preoperative radiologic examination included magnetic resonance imaging and standing plain radiographs. Surgery time, length, and diameter of femoral and tibial sockets and operative observations of accompanied meniscal and cartilage pathology were recorded using the IKDC surgical documentation form. We also separated patients in each group as having a low diameter graft (measured 7.5 or $8 \mathrm{~mm}$ ) and a high diameter graft $(\geq 8.5 \mathrm{~mm})$. Patients with graft diameter $<7.5$ mm were excluded $(n=3)$ from the study.

Postoperatively, the primary outcome measures were (1) anteroposterior knee stability at 2 years' follow-up measured using the KT-1000 knee instrumented laxity measuring device (MEDmetric Corp, San Diego, CA) and (2) femoral and tibial tunnels' widening measured using CT imaging performed at 12 months in comparison with the postoperative values performed within the first 48 hours postsurgery. These measurements were calculated according to the technique suggested by Sabat et al. ${ }^{2}$ at the coronal and sagittal CT sections, after defining 3 points in both the femoral $(\mathrm{F})$ and tibial $(\mathrm{T})$ tunnels: (1) the aperture point ( $\mathrm{Fl}$ and $\mathrm{Tl}$ ), (2) the furthest point (proximal suspension point in femur [F3] and distal point in tibia [T3]) and (3) the point between the aperture and the furthest where the diameter was maximum (midway point) (F2 and T2). CT image acquisition was performed on a Toshiba Aquilion PRIME, $80 \times$ multidetector dual-energy scanner (Toshiba Medical Systems Corporation, Tokyo, Japan). Patients were scanned supine, with the affected knee joint in neutral position. The study protocol 


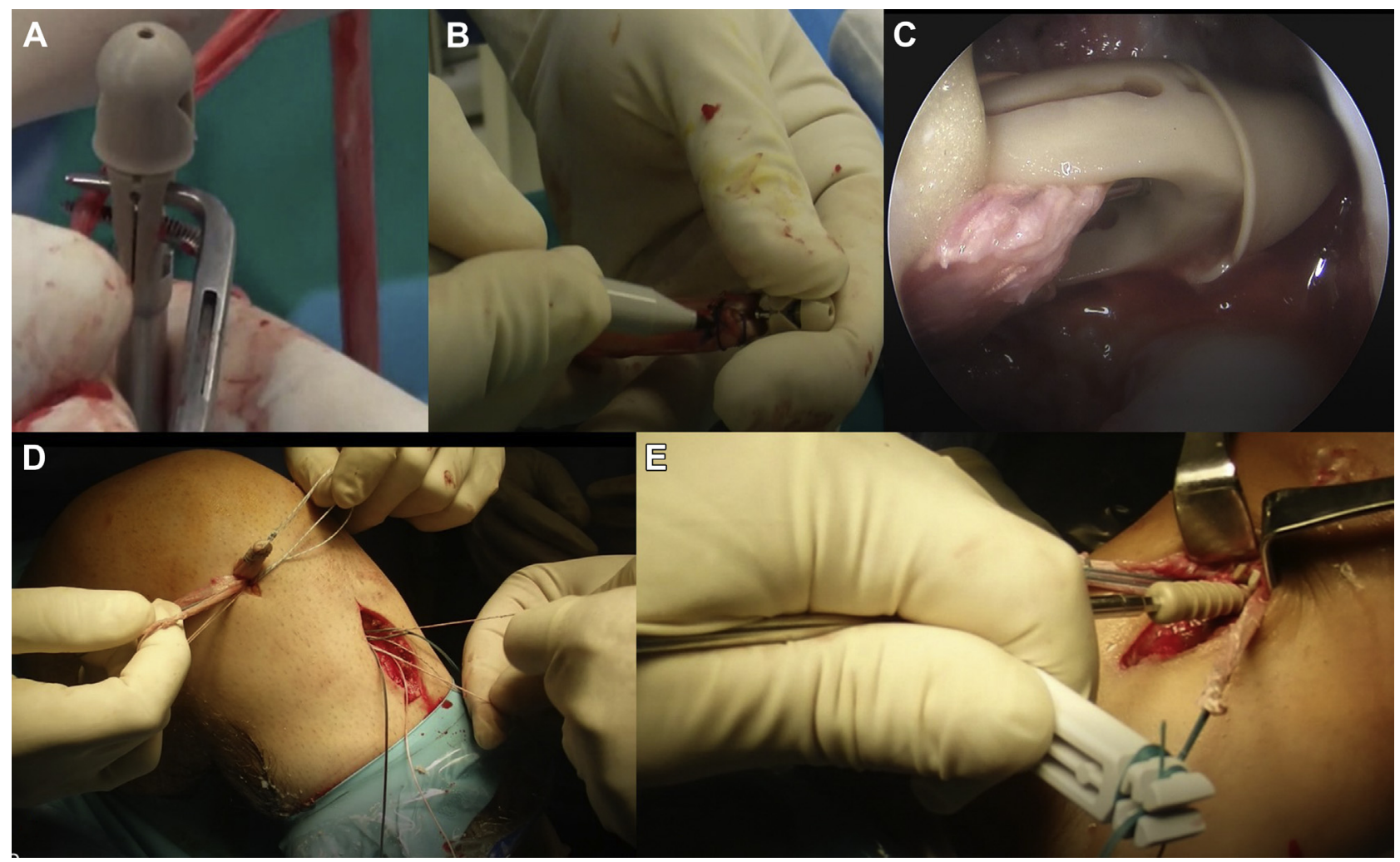

Fig 1. Intraoperative details of the expandable implantation in a right knee. (A) Passage of the hamstring tendons through the eyelet of the femoral AperFix device. (B) Marking of the aperture depth indicator to ensure proper femoral fixation at joint line. (C) Arthroscopic picture just before the insertion of AperFix femoral implant. (D) Intraoperative picture showing the gradual retrieval of each tendon (whipstitched with different suture) while the assistant is holding the rest of them and (E) fixation at the tibial site using the AperFix sheath and screw tibial implant.

consisted of primary source axial images obtained craniocaudally, using the single-energy metal artifact reduction algorithm. Image acquisition parameters were $135 \mathrm{kVp}$ and $300 \mathrm{mAs}$. The field of view was set at $170 \mathrm{~mm}$, slice thickness $0.5 \mathrm{~mm}$ with collimation 0.5 $\mathrm{mm} \times 160$. Images were reconstructed in bone and soft-tissue algorithm.

\section{Secondary Outcome Measures}

Secondary outcome measures included (1) pain level on the 1st, 2nd, 7th, and 15th postoperative day using the visual analog scale pain scale from 1 to 10 , with 0 representing the complete absence of pain and (2) the subjective IKDC 2000 assessment form, the Lysholm score and the Tegner activity scale at 3, 6, 12, and 24 months' postsurgery. All preoperative and postoperative clinical scores and KT-1000 measurements were performed by a certified physiotherapist not involved initially with the study.

\section{Surgical Technique, Medication, and Rehabilitation}

All patients underwent inpatient ACL reconstruction under general anesthesia with the use of 4-stranded (quadruple) hamstring autograft. All grafts were rinsed with hydrogen peroxide and soaked in vancomycin solution before their insertion. Three doses of second-generation cephalosporin were administered for chemoprophylaxis, and a low-molecular-weight heparin was prescribed for 3 weeks for thromboprophylaxis. Hamstring tendons were received without the use of tourniquet to avoid muscle contraction and graft strain but the tourniquet was inflated to $350 \mathrm{~mm} \mathrm{Hg}$ during the arthroscopic procedure. Femoral sockets were created in both groups with the knee in $120^{\circ}$ of flexion, through an accessory medial portal, after marking the anatomical footprint of the ACL with a spinal needle.

In group $\mathrm{A}$, femoral fixation was performed in a standard fashion using fixed-looped buttons (from Stryker, Biomet, or DePuy companies). The length of the cradle was $25 \mathrm{~mm}$ in 18 cases and $20 \mathrm{~mm}$ in 6. In group B, the AperFix AM femoral implant with a standard length of $24 \mathrm{~mm}$ (Cayenne Medical, Inc., Scottsdale, AZ) was used through the anteromedial portal; the length of the femoral socket was always $25 \mathrm{~mm}$. The size of the implant (diameter 9,10 , or 11 ) was determined by the graft diameter $(7.5,8-9$, or $9-10$ in respect). The prepared hamstring tendons were loaded to the separate eyelets of the implant, whipstitched with sutures of different color, and their suture ends were wrapped 

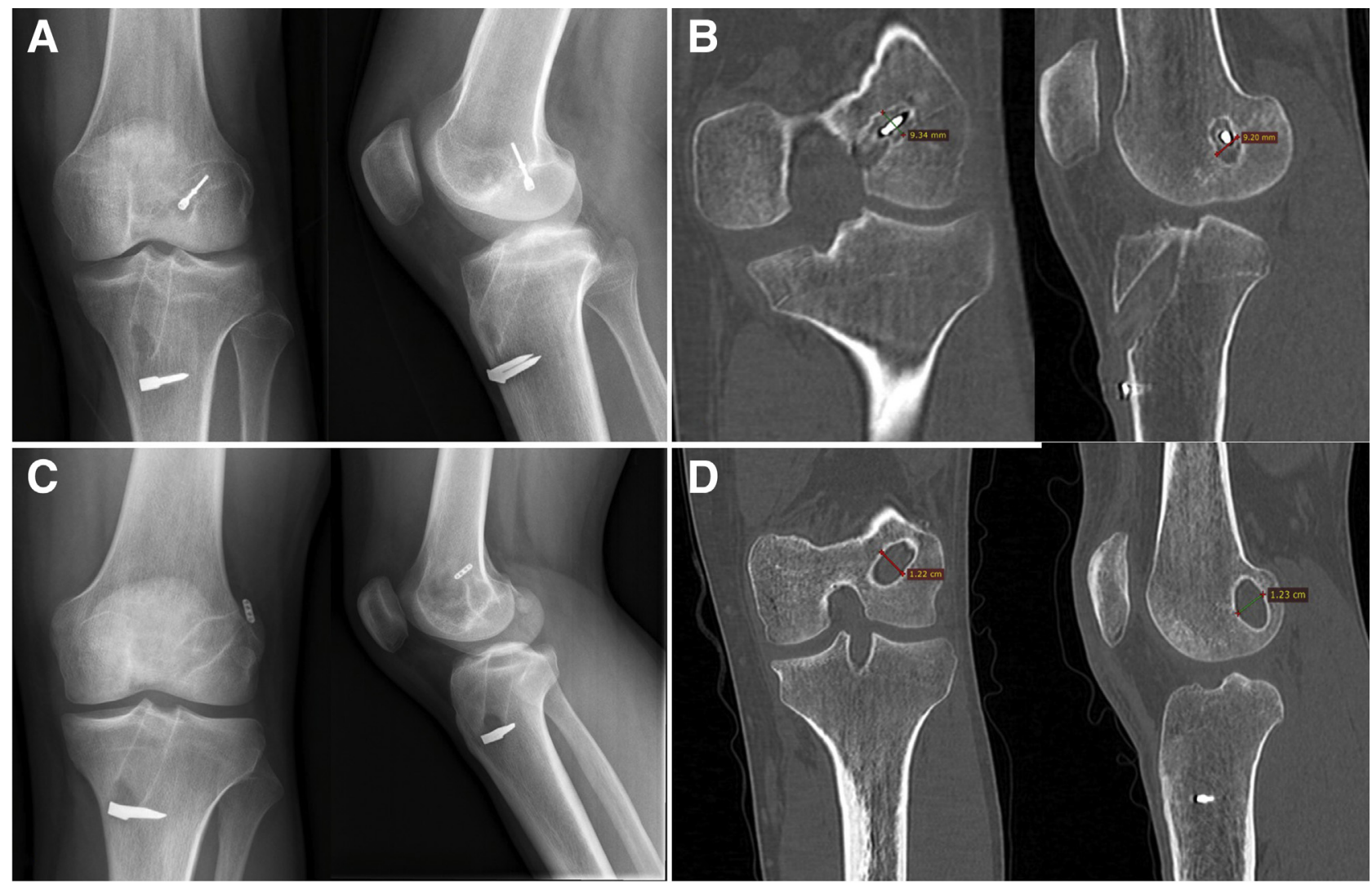

Fig 2. (A) Anteroposterior and lateral radiographs of a 22 -year-old male patient from the expandable group (left knee) showing good positioning of the implants, and (B) measurement of femoral tunnel widening in sagittal and coronal CT images at the midpoint (F2). (C) Anteroposterior and lateral radiographs of a 22-year-old male patient from the cortical button group (left knee) showing good positioning of the implants. (D) Measurement of femoral tunnel widening in sagittal and coronal CT images at the midpoint (F2). (CT, computed tomography.)

around the suture cleats of the femoral inserter handle in a fashion that resembles a "double-bundle ACL reconstruction through singe tunnel" (Fig 1A). The accessory medial portal was extended by $2 \mathrm{~cm}$ to allow the passage of the implant and a looped recovery suture was passed inside the joint and retrieved from the tibial tunnel while both its ends were secured with forceps. Graft preparation was completed, after drawing a horizontal mark on the top of the tendon bundles at the inferior edge of the implant to serve as the aperture depth indicator (Fig 1B). The implant was inserted through the accessory medial portal towards the femoral socket with the knee in $120^{\circ}$ of flexion and was impacted until the horizontal mark was flush with the femur (Fig 1C). Deployment of the screw was performed to compress the graft against the tunnel walls and the inserter handle from the implant was released with caution by pulling back on the implant release knob. The anchor's stability was verified by pulling the sutures from the medial portal. After completion of femoral fixation, we proceeded with the passage of the tendons through the knee joint; the sutures of both ends of the tendons were passed through the loop of the recovery suture and pulled down into the tibial tunnel, while holding the 4 tendons around the assistant's finger or using forceps, so that they did not pass all together at once (Fig 1D). After passing all 4 sutures from the tibial tunnel one tendon at a time was gradually recovered, pulling the corresponding suture while holding the rest, until all 4 tendons finally were passed through the tibial tunnel.

After performing 10 to 15 cycles of flexion-extension to calibrate the graft's tension, we proceeded with the tibial fixation (Fig 1E). The implant used in both groups was the AperFix II Tibial Implant with Driver system (Cayenne Medical, Inc.) using screws $1 \mathrm{~mm}$ larger than the diameter of the tibial tunnel. The graft was fixed in $15^{\circ}$ to $20^{\circ}$ of flexion and an additional staple was used in all cases (Fig 1D). At the completion of the surgical procedure, the incision sites were infiltrated with $30 \mathrm{~mL}$ of $0.25 \%$ bupivacaine with epinephrine and a drainage was applied for 24 hours. Patients were discharged from the hospital wearing a hinged knee brace for 6 weeks, cold therapy instructions and painkillers on demand. Postoperative ACL rehabilitation was focused on active full extension, quadriceps isometric exercises and passive kinesiotherapy. Straight-ahead running was initiated at 3 months, cutting or pivoting at 6 months, and contact 
Table 1. Demographic Data of the 2 Groups

\begin{tabular}{|c|c|c|c|}
\hline Variable & Cortical Button $(\mathrm{N}=24)$ & AperFix $(\mathrm{N}=24)$ & $P$ Value \\
\hline$\overline{\text { Age, } y, \text { mean } \pm S D}$ & $27 \pm 7.5$ & $28 \pm 8.3$ & $P=.763$ \\
\hline Height, $\mathrm{cm}$, mean $\pm \mathrm{SD}$ & $1.77 \pm 0.07$ & $1.79 \pm 0.2$ & $P=.310$ \\
\hline Weight, $\mathrm{kg}$, mean $\pm \mathrm{SD}$ & $78.46 \pm 11.23$ & $81.34 \pm 13.33$ & $P=.752$ \\
\hline Surgery time, mean \pm SD & $68.33 \pm 4.92$ & $75.42 \pm 4.98$ & $P<.001$ \\
\hline \multicolumn{4}{|l|}{ Meniscal pathology } \\
\hline Normal, n (\%), yes & $14(58.3 \%)$ & $15(62.5 \%)$ & $P=.764$ \\
\hline Medial and lateral tear, $\mathrm{n}(\%)$, yes & $0(0 \%)$ & $1(4.2 \%)$ & $P=.313$ \\
\hline Posterior horn, n $(\%)$, yes & $3(12.5 \%)$ & $2(8.3 \%)$ & $P=.638$ \\
\hline \multicolumn{4}{|l|}{ Cartilage pathology } \\
\hline None, $\mathrm{n}(\%)$, yes & $20(83.3 \%)$ & $20(83.3 \%)$ & $P=1$ \\
\hline Patella, n $(\%)$, yes & $1(4.2 \%)$ & $0(0 \%)$ & $P=.313$ \\
\hline MFC, $\mathrm{n}(\%)$, yes & $2(8.3 \%)$ & $4(16.7 \%)$ & $P=.384$ \\
\hline
\end{tabular}

sporting activities at 9-12 months postoperatively. Data were collected and recorded at 3, 6, 12, and 24 months (for patient-reported outcome measures and anteroposterior knee stability) and at 12 months (for CT evaluation of tunnel widening) (Fig 2).

\section{Statistical Analysis}

Sample size analysis showed that a minimum sample of 24 patients per group was required to detect a clinically relevant side-to-side difference in knee anteroposterior laxity of $2.5 \mathrm{~mm}$ (compared with the normal, contralateral knee and based on KT-1000 measurement) between the 2 groups, assuming an SD of $3.0 \mathrm{~mm}$; for the power analysis, the a value was set at .05 and the $b$ value at $.8 .^{3} \mathrm{~A}$ total of 70 patients were included in the study; this would have resulted in a minimum of 24 patients in each group had 9 patients in each group $(<25 \%)$ been excluded due to the additional operations, refusal to perform CT at 1 year or lost to follow-up. Initially, all of the quantitative variables were examined for normality via the Shapiro-Wilk test. Regarding the descriptive statistics, the variables are described with mean \pm standard deviation. Regarding the inferential statistics, associations between group A (cortical button) or group B (AperFix) and each variable were examined either with Student $t$ test (the parametric scenario) or
Wilcoxon rank-sum test (the nonparametric scenario). The comparison of 2 population proportions was performed using the binomial z test. Statistical tests were considered as 2 -sided, and statistical significance was taken when $P<.05$. The statistical analysis was held using the R software for statistical computing, with the assistance of the RStudio interface (both open-source products).

\section{Results}

Twenty-four patients were available in each group for the final evaluation. There were no demographic differences between groups regarding age $(P=.763)$, height $(P=.310)$, weight $(P=.752)$, body mass index $(P=.821)$, and associated meniscal or cartilage pathology (Table 1). The mean surgical time was $68 \mathrm{mi}-$ nutes or the cortical button group versus 75 minutes for the expandable group $(P<.001)$. This difference can be explained by the additional time needed for the retrograde passage of the tendons from the medial portal to the tibia in the expandable group. The mean hamstring's graft diameter was $8.75 \pm 0.68$ for the cortical button group and $7.98 \pm 0.57$ for the expandable group $(P<.001)$. In the cortical button group, only 5 patients $(21 \%)$ had small diameter grafts (1 patient $7.5 \mathrm{~mm}$ and 4 patients $8 \mathrm{~mm}$ ) whereas in the expandable group 17

Table 2. KT-1000 Measurements

\begin{tabular}{lccc}
\hline \multicolumn{1}{c}{ KT-1000 Measurement } & Cortical Button & AperFix & $P$ Value \\
\hline Preoperative, mean \pm SD & $8.71 \pm 1.99$ & $9.23 \pm 2.48$ & $P=.471$ \\
3 months' follow-up, mean \pm SD & $7.27 \pm 1.62$ & $8.01 \pm 1.48$ & $P=.041$ \\
6 months' follow-up, mean \pm SD & $6.35 \pm 1.46$ & $6.73 \pm 1.07$ & $P=.141$ \\
12 months' follow-up, mean \pm SD & $5.79 \pm 1.65$ & $6.21 \pm 1.11$ & $P=.095$ \\
24 months' follow-up, mean \pm SD & $5.27 \pm 1.76$ & $5.63 \pm 1.42$ & $P=.310$ \\
\hline
\end{tabular}

SD, standard deviation. 
Table 3. Relative (\%) Femoral and Tibial Tunnel Widening in 2 Planes and 3 Points of Interest 12 Months Postoperatively Using CT in 2 Planes

\begin{tabular}{lccc}
\hline \multicolumn{1}{c}{ CT Coronal Plane } & Cortical Button $(\%)$ & AperFix $(\%)$ & $P$ Value \\
\hline Femur aperture F1 & $9.97 \pm 10.24$ & $11.21 \pm 7.34$ & $P=.411$ \\
Femur midway F2 & $13.03 \pm 12.68$ & $5.42 \pm 6.33$ & $P=.072$ \\
Femur distal point F3 & $8.09 \pm 11.47$ & $3.76 \pm 5.56$ & $P=.873$ \\
Tibia aperture T1 & $9.86 \pm 10.38$ & $7.63 \pm 7.66$ & $P=.677$ \\
Tibia midway T2 & $17.34 \pm 12.53$ & $8.19 \pm 7.6$ & $P=.065$ \\
Tibia distal point T3 & $15.65 \pm 11.34$ & $10.21 \pm 9.33$ & $P=.100$ \\
CT sagittal plane & & & $17.55 \pm 12.33$ \\
Femur aperture F1 & $11.2 \pm 9.34$ & $P=.068$ \\
Femur midway F2 & $13.08 \pm 10.77$ & $11.33 \pm 8.21$ & $P=.836$ \\
Femur distal point F3 & $8.87 \pm 13.9$ & $6.32 \pm 7.22$ & $P=.962$ \\
Tibia aperture T1 & $11.97 \pm 11.33$ & $5.52 \pm 8.21$ & $P=.033$ \\
Tibia midway T2 & $14.96 \pm 24.16$ & $8.11 \pm 10.45$ & $P=.377$ \\
Tibia distal point T3 & $11.58 \pm 11.83$ & $7.99 \pm 10.4$ & $P=.260$ \\
\hline CT, Compun
\end{tabular}

CT, computed tomography.

patients $(71 \%)$ had graft diameter of 7.5 (3 patients) or 8 (14 patients). The mean intraoperative femoral tunnel diameter was different between the 2 groups because expandable femoral implants had by definition a diameter of 9 or 10 (cortical button: $8.79 \pm 0.71$, expandable: $9.73 \pm 0.33, P<.001$ ); in the tibia, there was no statistical difference (cortical button $9.1 \pm 0.68$ vs expandable $8.58 \pm 0.38, P=.007)$. All patients were evaluated at least 24 months' postsurgery.

The first primary outcome, anteroposterior knee stability measured with the KT-1000 arthrometer, showed no difference between groups at 24 months' follow-up $(P=.31)$, as well as at 3,6 , and 12 months postoperatively (Table 2 ). The second primary outcome, the percent widening $(\%)$ of femoral and tibial tunnel at 1 year follow up, evaluated in coronal and sagittal CT planes, showed no difference also, except for greater values at the tibial coronal point T2 $(P=.065)$ and tibial sagittal point $\mathrm{Tl}(P=.033)$ in the group of cortical buttons. The percent (\%) femoral tunnel widening at any point of interest in both coronal and sagittal planes showed no statistical difference between the 2 groups (Table 3). After summing up these values in both planes, the mean widening of femoral tunnel showed an increase of $10.7 \%$ for the cortical button vs $9.24 \%$ for the expandable group whereas in the tibia the widening was $13.56 \%$ vs $7.88 \%$ in respect (Fig 2). Another parameter that we studied was the correlation of tendon graft diameter in each group with the final clinical and knee instrumented laxity (KT-1000) outcomes at 24 months of follow-up. There were no statistical differences between patients with low diameter graft $(7.5$ or 8$)$ versus high diameter grafts $(\geq 8.5)$ in both groups (Table 4).

Secondary clinical outcomes (IKDC subjective evaluation form, Lysholm score, and Tegner scale) showed no statistical difference between groups at 3, 6, 12, and 24 months' postoperatively (Table 5). Numerical pain scale (visual analog scale) was similar in both groups (Table 6) except for postoperative day 7, were the expandable group showed better results $(P=.014)$. There were no major intraoperative and late postoperative complications in any of the groups.

\section{Discussion}

The most important finding of the present study was that the clinical and radiologic results of arthroscopic ACL reconstruction with hamstrings autograft were not affected by the different femoral fixation devices we had used. Our main hypothesis of improved outcomes in the expandable group in terms of stability and tunnel enlargement at minimum 2 years' follow-up was not confirmed. There were also no statistically significant differences in mean continuous IKDC, Tegner, or Lysholm scores between expandable and cortical button groups. In the literature, there is no consensus regarding the optimal femoral fixation method in ACL reconstruction with soft tissue grafts. The most commonly used methods are cortical buttons (CB) or adjustable buttons, followed by cross-pins (CP) and

Table 4. Correlation of Graft Diameter With Clinical Outcome (IKDC, Lysholm, Tegner) and Knee Instrumented Laxity (KT-1000)

\begin{tabular}{|c|c|c|c|c|c|c|}
\hline $\begin{array}{l}\text { Variable (Preoperative and } \\
\text { at } 24 \text { Months' Follow-Up) }\end{array}$ & \multicolumn{3}{|c|}{ Cortical Button } & \multicolumn{3}{|c|}{ AperFix } \\
\hline IKDC 2000 preoperative & $74.28 \pm 9.69$ & $66.2 \pm 11.43$ & $P=.153$ & $61.04 \pm 10.23$ & $56.28 \pm 21.6$ & $P=.633$ \\
\hline IKDC 200024 mo & $98.36 \pm 2.4$ & $92.85 \pm 12.62$ & $P=.604$ & $97.37 \pm 3.72$ & $93.7 \pm 7.08$ & $P=.134$ \\
\hline Lysholm preoperative & $86 \pm 9.57$ & $82.32 \pm 11.28$ & $P=.484$ & $84.89 \pm 8.97$ & $82.17 \pm 13.72$ & $P=.066$ \\
\hline Tegner 24 mo & $8 \pm 1.41$ & $7.79 \pm 1.4$ & $P=.88$ & $8.22 \pm 1.23$ & $7.84 \pm 1.54$ & $P=.323$ \\
\hline KT 1000 preoperative & $8.9 \pm 2.41$ & $8.66 \pm 1.94$ & $P=.843$ & $9.01 \pm 1.88$ & $8.3 \pm 3.19$ & $P=.134$ \\
\hline KT $100024 \mathrm{mo}$ & $5.4 \pm 1.52$ & $5.24 \pm 1.86$ & $P=.844$ & $5.56 \pm 1.56$ & $5.35 \pm 1.76$ & $P=.262$ \\
\hline
\end{tabular}

IKDC, International Knee Documentation Committee. 
Table 5. Clinical Outcome in Both Groups At 3, 6, 12, and 24 Months Postoperatively

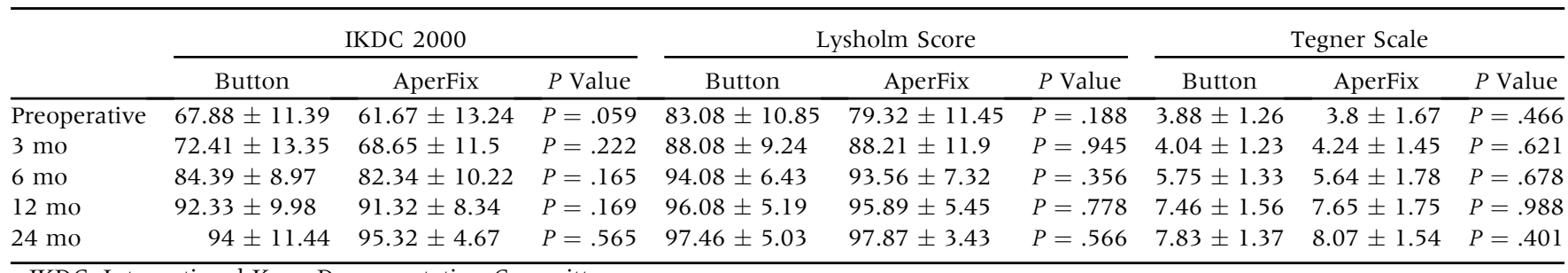

IKDC, International Knee Documentation Committee.

interference screws (IS), providing cortical, corticocancellous, and compressive cancellous anchoring in respect. In the most recent systematic reviews and network meta-analyses of randomized control studies, $^{22-24}$ there was no differences in failure rate, knee stability, functional outcomes, or revision rates, although Yan et al. ${ }^{23}$ reported that IS was more likely to perform better results in comparison with CB and CP.

Colvin et al., ${ }^{11}$ in a similar meta-analysis in 2011, showed similar postoperative functional outcomes of aperture fixation (IS) in comparison with other methods (away from the joint line), but there was a trend toward decreased risk of surgical failures (relative risk $=0.57$ ) for the IS. Similarly, Eysturoy et al. ${ }^{5}$ showed also that $\mathrm{CB}$ fixation has a relatively increased rate of revision rate (hazard ratio 1.24) in a study of 13,200 patients treated with either patella tendon graft $(\mathrm{n}=1405)$ or a hamstring graft $(\mathrm{n}=11795)$ using 4 different fixation methods: $\mathrm{CB}$ $(\mathrm{n}=4680)$, adjustable CB $(\mathrm{n}=577)$, CP $(\mathrm{n}=5921)$, and IS $(\mathrm{n}=617)$. In addition, Lee et al. ${ }^{12}$ showed greater widening of the femoral tunnel when using $\mathrm{CB}$ fixation than transfemoral cross-pin fixation. Finally, recent studies comparing fixed versus adjustable buttons have shown no significant differences in their clinical performance, stability, and tunnel widening. ${ }^{25,26}$

Considering all these controversies with CBs, being the implant of choice in our practice for many years, we hypothesized that a stronger aperture femoral device would lead to better clinical, radiologic, and stability outcomes in our ACL patients. The AperFix system has different biomechanical properties from an IS providing what is referred to as "expandable fixation," thus avoiding the insertional torque, which can theoretically

Table 6. VAS Pain Scale in Both Groups at 1st, 2nd, 7th, and 15th Postoperative Day

\begin{tabular}{lccc}
\hline VAS Score, mean \pm SD & Cortical Button & AperFix & $P$ Value \\
\hline Day 1 & $3.5 \pm 1.62$ & $3.24 \pm 1.88$ & $P=.365$ \\
Day 2 & $2.38 \pm 1.47$ & $1.87 \pm 1.65$ & $P=.074$ \\
Day 7 & $2.04 \pm 1.2$ & $1.15 \pm 1.75$ & $P=.014$ \\
Day 15 & $0.62 \pm 0.65$ & $0.78 \pm 1.49$ & $P=.164$ \\
\hline
\end{tabular}

SD, standard deviation; VAS, visual analog scale. damage the graft or change its position within the tunnel; it also provides circumferential graft compression rather than the unilateral compression of an IS. In addition, the design of the AperFix femoral implant facilitates the separation of the anteromedial and posterolateral bundles of the ACL graft, representing theoretically an equivalent of a "double-band ACL through a single tunnel."

Gadikota et al. ${ }^{19}$ evaluated the mechanical stability of the AperFix system using fresh-frozen human cadaveric knee specimens. They performed AperFix and singlebundle cortical fixed-looped ACL reconstruction and subsequently investigated the knee joint kinematics to an anterior tibial load (130 N), a simulated quadriceps load $(400 \mathrm{~N})$, and combined torques at various degrees of knee flexion using a robotic testing device. They found that the AperFix system can better restore anterior tibial translation to the intact level at low flexion angles $\left(<30^{\circ}\right)$ compared with CB fixation, and this was one of the reasons that we decided to investigate the clinical relevance of this finding. Regarding tibial side, the AperFix screw and sheath combination has been evaluated in biomechanical testing by Aga et al., ${ }^{27}$ who tested 8 different tibial intratunnel softtissue fixation devices in a porcine model with bovine tendons. The AperFix device had the third strongest ultimate failure load $(1122 \pm 182.9 \mathrm{~N})$ after GraftBolt and INTRAFIX and the second least amount of cyclic displacement after 1000 cycles $(1.58 \pm 0.21 \mathrm{~mm})$, after GraftBolt.

In our study population, we decided to use the same tibial fixation device in both groups and an anteromedial portal for the insertion of either the $\mathrm{CB}$ or the expandable femoral device. We consider that choosing a more anatomical femoral insertion we would obtain better results in terms of knee stability. Mulcahey et al. ${ }^{28}$ compared transtibial and anteromedial insertion of AperFix system and found a significant improvement in IKDC scores in the anteromedial cohort but no significant differences in postoperative KT-1000 measurements between transtibial $(1.571 \pm 0.22 \mathrm{~mm})$ and anteromedial cohort $(1.246 \pm 0.09 \mathrm{~mm})$. Ozel et al. ${ }^{21}$ in contrast, reported significantly greater femoral tunnel width and anterior knee translation in the transtibial group (42 patients) in comparison with anteromedial 
group (38 patients) in a mean follow up period of 40.7 months; Lysholm and Tegner scores were not significantly different between the 2 groups. In this study, femoral tunnel widening was measured by comparing postoperative radiographs with final follow-up radiographs.

The overall clinical performance of the AperFix system in comparative studies with other types of femoral fixation is still inconsistent. Uzumcugil et al. ${ }^{17}$ compared the clinical outcomes of AperFix vs Transfix in a retrospective comparative study of 38 patients. There was no significance difference between the 2 groups in terms of laxity testing and complication rates except from a small but apparent difference between the 2 fixation techniques in terms of range of movement (by $10^{\circ}$ in favor of TransFix) and Lysholm scores (by 6 points in favor of AperFix). Aydin and Ozcan ${ }^{18}$ compared 3 different femoral fixation devices in a cohort of 100 patients (AperFix: 31, Transfix: 35, and ENDOBUTTON: 34 ) and found no significant difference between these devices in terms of clinical symptoms, findings and scoring systems evaluated. In contrast, Eajazi et al. $^{20}$ in a randomized comparative study of AperFix, RIGIDFIX, and ENDOBUTTON in 120 patients (40 in each group) found AperFix to yield better improvement of Lysholm score compared with RIGIDFIX, and the 2 of them yielded better results (in terms of improvement in Lysholm score) compared with ENDOBUTTON. Re-examination of patients 2 years after surgery revealed 6 cases of failure in the ENDOBUTTON group, with failure defined as an anterior tibial displacement of more than $7 \mathrm{~mm}$ using KT-1000. There were 4 cases of failure in the RIGIDFIX group, whereas only one case of failure was observed in the AperFix group. Finally, another study from Uzumcugil et al. ${ }^{16}$ comparing AperFix $(n=18)$, TransFix $(n=29)$, and ENDOBUTTON $(n=20)$ in a nonrandomized fashion resulted that tunnel widening between groups was not significantly different in terms of coronal and sagittal femoral tunnel diameters. Tibial tunnel diameter increase in the sagittal plane in the ENDOBUTTON group was considerably smaller than in the TransFix and AperFix groups. In our study, the results were different, with the CB group having significant differences in tibial tunnel enlargement in both planes. A possible explanation of these differences from Uzumcugil et al. ${ }^{16}$ is that they used IS for tibial fixation in the TransFix and ENDOBUTTON groups and that they had different evaluation method of tunnel enlargement using also plain radiographs instead of CT scans. If someone summarizes the results of these studies, he or she can assume that the performance of the AperFix is at least similar to other commonly used devices.

In our study, the expandable system shown equal results to fixed-looped CB but its theoretical advantages of stronger aperture fixation, less tunnel widening and "double-bundle ACL" assimilation were not reflected to the final outcome scores, anteroposterior knee stability and the degree of tunnel enlargement. Because of the rigidness of the femoral implant, that requires special instrumentation for implant removal, there is concern among surgeons, regarding the difficulties and appropriateness of special instrumentation in case of revision surgery. Cooper et al. ${ }^{29}$ were the first authors reported a successful removal of an AperFix femoral implant using the specific manufacturers instrumentation without any associated morbidity to either the femoral or tibial tunnels. The main strengths of our study are (1) the prospective randomized design, (2) the exclusive inclusion of male patients with similar demographic data and minimal meniscal and cartilage pathology, (3) the proper radiological evaluation with CT scan (in 2 planes), and (4) the exclusion of patients that underwent additional operations, missed the 1 -year CT appointment or had graft diameters $<7.5 \mathrm{~mm}$.

\section{Limitations}

Weaknesses can be considered in our study: (1) the small final number of patients in each group but this was a homogenous population with minimal associated pathology and consistent with our lower requirements of power analysis; (2) the nonvolumetric tunnel widening evaluation that was performed by only one radiologist, using the ruler application in the Centricity Enterprise Web V2.1 PACS viewing system; and (3) the relative short period (2 years) of the last follow up evaluation.

\section{Conclusions}

Our results showed no significant differences in knee anteroposterior stability, tunnel enlargement, or other clinical outcomes comparing expandable versus $\mathrm{CB}$ fixation in anteromedial hamstring ACL reconstruction.

\section{References}

1. Fauno P, Kaalund S. Tunnel widening after hamstring anterior cruciate ligament reconstruction is influenced by the type of graft fixation used: A prospective randomized study. Arthroscopy 2005;21:1337-1341.

2. Sabat D, Kundu K, Arora S, Kumar V. Tunnel widening after anterior cruciate ligament reconstruction: a prospective randomized computed tomography—based study comparing 2 different femoral fixation methods for hamstring graft. Arthroscopy 201 1;27:776-783.

3. Lubowitz JH, Schwartzberg R, Smith P. Cortical suspensory button versus aperture interference screw fixation for knee anterior cruciate ligament soft-tissue allograft: A prospective, randomized controlled trial. Arthroscopy 2015;31:1733-1739.

4. Monaco E, Fabbri M, Redler A, et al. Anterior cruciate ligament reconstruction is associated with greater tibial tunnel widening when using a bioabsorbable screw compared to an all-inside technique with suspensory 
fixation. Knee Surg Sports Traumatol Arthrosc 2019;(27): 2577-2584.

5. Eysturoy NH, Nissen KA, Nielsen T, Lind M. The influence of graft fixation methods on revision rates after primary anterior cruciate ligament reconstruction. Am J Sports Med 2018;46:524-530.

6. Ilahi OA, Nolla JM, Ho DM. Intra-tunnel fixation versus extra-tunnel fixation of hamstring anterior cruciate ligament reconstruction: A meta-analysis. J Knee Surg 2009;22:120-129.

7. Saccomanno MF, Shin JJ, Mascarenhas R, et al. Clinical and functional outcomes after anterior cruciate ligament reconstruction using cortical button fixation versus transfemoral uspensory fixation: A systematic review of randomized controlled trials. Arthroscopy 2014;30:1491-1498.

8. Jiang H, Ma G, Li Q, Hu Y, Li J, Tang X. Cortical button versus cross-pin femoral fixation for hamstring anterior cruciate ligament reconstruction: A meta-analysis of randomized controlled trials. Am J Sports Med 2018;46: 2277-2284.

9. Hu B, Shen W, Zhou C, Meng J, Wu H, Yan S. Cross pin versus interference screw for femoral graft fixation in hamstring anterior cruciate ligament reconstruction: A systematic review and meta-analysis of clinical outcomes. Arthroscopy 2018;34:615-623.

10. Browning WM 3rd, Kluczynski MA, Curatolo C, Marzo JM. Suspensory versus aperture fixation of a quadrupled hamstring tendon autograft in anterior cruciate ligament reconstruction: A meta-analysis. Am J Sports Med 2017;45:2418-2427.

11. Colvin A, Sharma C, Parides M, Glashow J. What is the best femoral fixation of hamstring autografts in anterior cruciate ligament reconstruction? A meta-analysis. Clin Orthop Relat Res 2011;469:1075-1081.

12. Lee DH, Son DW, Seo YR, Lee IG. Comparison of femoral tunnel widening after anterior cruciate ligament reconstruction using cortical button fixation versus transfemoral cross-pin fixation: a systematic review and meta-analysis. Knee Surg Relat Res 2020;32:11.

13. Ehrensberger M, Hohman DW Jr, Duncan K, Howard C, Bisson L. Biomechanical comparison of femoral fixation devices for anterior cruciate ligament reconstruction using a novel testing method. Clin Biomech (Bristol, Avon) 2013;28:193-198.

14. Görmeli G, Görmeli CA, Karakaplan M, Korkmaz MF, Diliçıkık U, Gözükara H. Outcome of transtibial AperFix system in anterior cruciate ligament injuries. Indian $J$ Orthop 2015:49:150-154.

15. Uribe JW, Arango D, Frank J, Kiebzak GM. Two-year outcome with the AperFix system for ACL reconstruction. Orthopedics 2013;36:e159-e164.

16. Uzumcugil O, Yalcinkaya M, Ozturkmen Y, Dikmen G, Caniklioglu M. Effect of PEEK polymer on tunnel widening after hamstring ACL reconstruction. Orthopedics 2012;35: e654-e659.
17. Uzumcugil O, Dogan A, Dalyaman E, et al. AperFix versus transfix in reconstruction of anterior cruciate ligament. J Knee Surg 2010;23:29-35.

18. Aydin D, Ozcan M. Evaluation and comparison of clinical results of femoral fixation devices in arthroscopic anterior cruciate ligament reconstruction. Knee 2016;23:227-232.

19. Gadikota HR, Wu JL, Seon JK, Sutton K, Gill TJ, Li G. Single-tunnel double-bundle anterior cruciate ligament reconstruction with anatomical placement of hamstring tendon graft: can it restore normal knee joint kinematics? Am J Sports Med 2010;38:713-720.

20. Eajazi A, Madadi F, Madadi F, Boreiri M. Comparison of different methods of femoral fixation anterior cruciate ligament reconstruction. Acta Med Iran 2013;51:444-448.

21. Ozel O, Yucel B, Orman O, Demircay E, Mutlu S. Comparison of anteromedial and transtibial ACL reconstruction using expandable fixation. Orthopedics 2017;40: e532-e537.

22. Hurley ET, Gianakos AL, Anil U, Strauss EJ, GonzalezLomas G. No difference in outcomes between femoral fixation methods with hamstring autograft in anterior cruciate ligament reconstruction-A network meta-analysis. Knee 2019:26:292-301.

23. Yan L, Li JJ, Zhu Y, et al. Interference screws are more likely to perform better than cortical button and cross-pin fixation for hamstring autograft in ACL reconstruction: A Bayesian network meta-analysis. Knee Surg Sports Traumatol Arthrosc 2021;29:1850-1861.

24. Singh S, Shaunak S, Shaw SCK, Anderson JL, Mandalia V. Adjustable loop femoral cortical suspension devices for anterior cruciate ligament reconstruction: A systematic review. Indian J Orthop 2020;54:426-443.

25. Ahn HW, Seon JK, Song EK, Park CJ, Lim HA. Comparison of clinical and radiologic outcomes and second-look arthroscopic findings after anterior cruciate ligament reconstruction using fixed and adjustable loop cortical suspension devices. Arthroscopy 2019;35:1736-1742.

26. Choi NH, Yang BS, Victoroff BN. Clinical and radiological outcomes after hamstring anterior cruciate ligament reconstructions: Comparison between fixed-loop and adjustable-loop cortical suspension devices. Am J Sports Med 2017;45:826-831.

27. Aga C, Rasmussen MT, Smith SD, et al. Biomechanical comparison of interference screws and combination screw and sheath devices for soft tissue anterior cruciate ligament reconstruction on the tibial side. Am J Sports Med 2013;41:841-848.

28. Mulcahey MK, David TS, Epstein DM, Alaia MJ, Montgomery KD. Transtibial versus anteromedial portal anterior cruciate ligament reconstruction using soft-tissue graft and expandable fixation. Arthroscopy 2014;30: $1461-1467$.

29. Cooper W, Machen MS, Nelson J, Owens BD. Anterior cruciate ligament revision of a relatively new implant system. Orthopedics 2009;32:326. 